\title{
SOLUTION OF THE WARING-GOLDBACH PROBLEM FOR ALGEBRAIC NUMBER FIELDS ${ }^{1}$
}

\author{
BY G. J. RIEGER
}

Communicated by I. J. Schoenberg, January 19, 1962

Let $P$ be the field of the rational numbers and $K$ an arbitrary algebraic number field.

1. Hua's solution for $P$. Let us denote by $k$ and $s$ arbitrary natural numbers, by $c(*)$ a positive constant depending on $*$ only, by $\mathfrak{P}^{k}$ the sequence consisting of 1 and the $k$ th powers of all rational prime numbers, by $s \mathfrak{F}^{k}$ the $s$-fold Schnirelmann sum $\mathfrak{P}^{k}+\cdots+\mathfrak{P}^{k}$, by $d\left(s \mathfrak{B}^{k}\right)$ the Schnirelmann density of $s \mathfrak{P}^{k}$, and by $I_{k, 8}(m)$ the number of solutions of

$$
p_{1}^{k}+\cdots+p_{s}^{k}=m, \quad p_{j} \in \mathfrak{P}^{1} \cup\{0\} \quad(j=1, \cdots, s) .
$$

The Waring-Goldbach problem for $P$ was solved by Hua in 1938 since he proved

THEOREM (1P). For every natural number $k$ there exists a $c_{1}(k)$ such that for every $s \geqq c_{1}(k)$ and every natural number $m$ we have $I_{k, s}(m)>0$.

Hua also proved the following stronger

THEOREM (2P). For

$$
s \geqq \begin{cases}2^{k}+1 & \text { in case } 1 \leqq k \leqq 10, \\ k^{2}(4 \log k+2 \log \log k+5) & \text { in case } k>10\end{cases}
$$

we have

$$
I_{k, 8}(m)=c_{2}(m) \frac{\Gamma^{s}\left(\frac{1}{k}\right) m^{s / k-1}}{\Gamma\left(\frac{s}{k}\right) \log ^{s} m}\left(1+O\left(\frac{\log \log m}{\log m}\right)\right)
$$

and (for the "singular series" $c_{2}(m)$ )

$$
\begin{aligned}
& c_{2}(m)<c_{3}, \\
& c_{2}(m)>c_{4},
\end{aligned}
$$

1 This work, carried out while the author was on leave at the University of Munich, Germany, was supported by the National Science Foundation grant G-16305 to Purdue University. 
with absolute positive constants $c_{3}$ and $c_{4}$.

The proof of (1) rests mainly on

(P1) the obvious formula

$$
I_{k, 8}(m)=\int_{0}^{1}\left(\sum_{p \leq m 1 / k} e^{2 \pi i p^{k} \alpha}\right)^{s} e^{-2 \pi i m \alpha} d \alpha \quad[2, p .93],
$$

(P2) the Farey decomposition of the path of integration (basic and supplementary intervals) [2, pp. 84-85],

(P3) an estimate of trigonometrical sums [2, Hilfssatz 7. 13],

(P4) an estimate of trigonometrical sums with primes [2, Satz 10],

(P5) the prime number theorem of Siegel and Walfisz [2, Hilfssatz 7. 14],

(P6) an estimate of complete trigonometrical sums [2, Satz 1]. In the proof of (1), the facts (P3) and (P4) are used to show that the contribution of the integral over the supplementary intervals is contained in the remainder term of (1); (P5) and (P6) are used to show that the contribution of the integral over the basic intervals yields the main term and the remainder term of (1). The proof of (2) uses only

(P7) simple facts on congruences.

The proof of (3) requires besides ( $\mathrm{P} 7)$ also

(P8) a (considerably deeper) theorem on the solvability of

$$
x_{1}^{k}+\cdots+x_{s}^{k} \equiv m \bmod p^{t}, p \nmid x_{1} \cdots x_{s} .
$$

(1) and (2) imply

$$
I_{k, 8}(m)<c_{5}(k, s) m^{8 / k-1} \log ^{-8} m .
$$

(4), (P5), and the Schwarz inequality give immediately (the classical idea of Schnirelmann [5])

Theorem (3P). For $s \geqq c_{6}(k)$ we have $d\left(s \mathfrak{P}^{k}\right)>0$.

Theorem (3P) is equivalent to Theorem (1P).

2. The solution for arbitrary $K$. Let us denote by $\tilde{\mathfrak{P}}^{k}$ the sequence consisting of 1 and the $k$ th powers of all totally positive prime numbers $\varpi$ of $K$, by $s \tilde{\mathfrak{H}}^{k}$ the generalized $s$-fold Schnirelmann sum $\tilde{\mathfrak{F}}^{k}+\cdots+\tilde{\mathfrak{F}}^{k}[4,(15)]$, by $d\left(s \tilde{\mathfrak{P}}^{k}\right)$ the generalized Schnirelmann density of $s \widetilde{\mathfrak{P}}^{k}[4,(16)]$, and by $J_{k, e}(\mu)$ for $\mu \in K$ the number of solutions of

$$
\tilde{\omega}_{1}^{k}+\cdots+\tilde{\omega}_{s}^{k}=\mu, \quad \varpi_{j} \in \tilde{\mathfrak{B}}^{1} \cup\{0\} \quad(j=1, \cdots, s) .
$$


Theorem (3K). For $s \geqq c_{7}(k, K)$ we have $d\left(s \tilde{\mathfrak{P}}^{k}\right)>0$.

The idea of the proof of Theorem $(3 \mathrm{~K})$ is the same as for Theorem (3P); one needs direct generalizations (K1), . , (K7) of (P1), . , (P7) for $P$ to $K$. Now, (K1) resp. (K2) resp. (K3) resp. (K5) resp. (K6) can be found in [6] resp. [6] resp. [4] resp. [3] resp. [1]. (K4) and (K7) are practically straightforward generalizations of (P4) and (P7). This completes the outline of a proof of Theorem (3K).

(K8) can also be obtained and one arrives at a Theorem (2K) for $J_{k, s}(\mu)$ which is too involved to be stated here.

A generalization to polynomials presents no serious difficulty.

I would like to express my gratitude to Professor Hans Rademacher for drawing my attention to [3].

\section{BIBLIOGRAPHY}

1. L. K. Hua, On exponential sums over an algebraic number field, Canad. J. Math. 3 (1951), 44-51.

2. - Additive Primzahltheorie, Teubner-Verlag, Leipzig, 1959.

3. T. Mitsui, Generalized prime number theorem, Jap. J. Math. 26 (1956), 1-42.

4. G. J. Rieger, Elementare Loesung des Waringschen Problems fuer algebraische Zahlkoerper mit der verallgemeinerten Linnikschen Methode, Math. Ann. (to appear).

5. L. G. Schnirelmann, Ueber additive Eigenschaften von Zahlen, Math. Ann. 107 (1933), 649-690.

6. C. L. Siegel, Generalization of Waring's problem to algebraic number fields, Amer. J. Math. 66 (1944), 122-136.

PuRdue University AND UNIVERSITY OF MUNICH 